ARTIGO ORIGINAL

\title{
Efeito do uso de meia elástica sobre os níveis dos biomarcadores de lesão muscular em atletas de voleibol após atividade física
}

\author{
Effect of elastic stockings on biomarkers levels of muscle soreness in volleyball players after exercise
}

Marcondes Figueiredo', Matheus Fidélis Figueiredo², Nilson Penha-Silva ${ }^{3}$

\begin{abstract}
Resumo
Objetivo: Avaliar os níveis plasmáticos dos biomarcadores de lesão muscular, a saber, creatina quinase, lactato desidrogenase e mioglobina, em atletas profissionais de voleibol após exercícios musculares anaeróbicos, com e sem uso de meia elástica.

Métodos: Foram avaliadas dez jogadoras profissionais de voleibol, com idades entre 18 e 25 anos, utilizando ou não meia elástica (Sport Active ${ }^{\circledR}$, Venosan, Abreu e Lima, Brasil), com compressão de 20 a 30 mmHg abaixo do joelho. As dosagens foram feitas em três momentos: M0, início da manhã, com as atletas em repouso sem uso de meia elástica; $M 1$, início da manhã, após a realização de atividade física com uso de meia elástica; $M 2$, sete dias depois, após a realização dos mesmos exercícios, porém sem uso de meia elástica. Aplicou-se a escala de Borg após cada série de atividade física para avaliação do esforço.

Resultados: Os valores médios obtidos para creatina quinase e lactato desidrogenase foram, respectivamente, de 117,7 $\pm 40,2$ e 134,2 $\pm 11,3 \mathrm{U} / \mathrm{L}$, em M0;

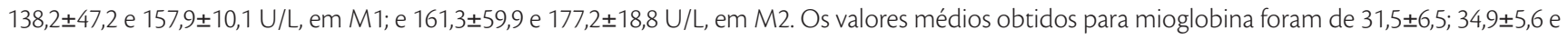
$38,6 \pm 12,6 \mu \mathrm{g} / \mathrm{L}$ nos momentos M0, M1 e M2, respectivamente. Houve diferenças estatisticamente significativas (Tukey) entre M1 e M2 para os valores de creatina quinase $(p=0,0007)$ e lactato desidrogenase $(p=0,000)$, mas não para os valores de mioglobina ( $p=0,1135)$. Os escores da escala de Borg em M1 e M2 foram, respectivamente, de 17,8 e 18,2, sem diferença estatisticamente significante entre eles (Wilcoxon).

Conclusão: $\mathrm{O}$ uso da meia elástica foi associado a menores níveis plasmáticos dos biomarcadores de lesão muscular após exercício físico.
\end{abstract}

Palavras-chave: meias de compressão; desempenho atlético; atividade física.

\begin{abstract}
Objective: To assess plasma levels of muscle soreness biomarkers, namely creatine kinase, lactate dehydrogenase, and myoglobin, in professional volleyball players following anaerobic exercise with and without the use of elastic stockings.

Methods: Ten female volleyball players aged 18 to 25 years-old were assessed with and without below-knee 20 to $30 \mathrm{mmHg}$ compression stockings (Sport Active ${ }^{\circledR}$, Venosan, Abreu e Lima, Brazil). Biomarker levels were assessed at three different moments: M0, early in the morning, with the athletes at rest, not using elastic stockings; M1, early in the morning, following a bout of exercise using elastic stockings; M2, seven days later, following the same bout of exercise, however not using elastic stockings. The Borg scale was used after each series of exercise to evaluate the physical effort.

Results: The means values obtained for creatine kinase and lactate dehydrogenase were, respectively, $117.7 \pm 40.2$ and $134.2 \pm 11.3 \mathrm{U} / \mathrm{L}$ at $\mathrm{MO}$; $138.2 \pm 47.2$ and $157.9 \pm 10.1 \mathrm{U} / \mathrm{L}$ at M1; and $161.3 \pm 59.9$ and $177.2 \pm 18.8 \mathrm{U} / \mathrm{L}$ at $\mathrm{M} 2$. The mean values obtained for myoglobin were $31.5 \pm 6.5 ; 34.9 \pm 5.6$ and $38.6 \pm 12.6 \mu \mathrm{g} / \mathrm{L}$ at the moments $\mathrm{MO}, \mathrm{M} 1$ and $M 2$, respectively. Statistically significant differences were observed between $M 1$ and $M 2$ (Tukey) for creatine kinase $(p=0.0007)$ and lactate dehydrogenase $(p=0.000)$, but not for myoglobin $(p=0.1135)$. Borg scale scores obtained at $M 1$ and $M 2$ were, respectively, 17.8 and 18.2, without statistically significant differences between them (Wilcoxon).

Conclusion: The use of elastic stockings was associated with lower plasma levels of biomarkers of muscle injury after exercise.
\end{abstract}

Keywords: elastic stockings; athletic performance; motor activity.

\footnotetext{
${ }_{1}^{1}$ Angiologia e Cirurgia Vascular, Universidade Federal de São Paulo (UNIFESP) - São Paulo (SP), Brasil.

${ }^{2}$ Faculdade de Medicina da Universidade Federal de Uberlândia (UFU) - Uberlândia (MG), Brasil.

${ }^{3}$ Instituto de Genética e Bioquímica da UFU - Uberlândia (MG), Brasil.

Fonte de financiamento: nenhuma.

Conflito de interesses: nada a declarar.

Submetido em: 30.03.11. Aceito em: 22.08.11.

J Vasc Bras. 2011;10(4):289-292.
} 


\section{Introdução}

O uso da meia elástica está bem estabelecido na prática clínica, no contexto da medicina baseada em evidências, tanto para profilaxia quanto para o tratamento de patologias linfáticas e venosas ${ }^{1}$. Além disso, o efeito benéfico das meias elásticas já atingiu a prática esportiva, proporcionando mais conforto aos praticantes de algumas modalidades ${ }^{2,3}$. No contexto esportivo, o principal objetivo do uso da meia é melhorar o desempenho do atleta e proporcionar boa recuperação após o esforço físico.

Em relação ao uso da meia elástica na prática esportiva, a literatura sugere um benefício indireto, com menores níveis de ácido lático pós-atividade, redução de trauma muscular e melhora no desempenho e no retorno venoso ${ }^{4-10}$.

O objetivo deste estudo foi avaliar os níveis plasmáticos dos biomarcadores de lesão muscular, a saber, creatina quinase $(\mathrm{CK})$, lactato desidrogenase $(\mathrm{LDH})$ e mioglobina $(\mathrm{MB})$, em atletas profissionais de voleibol após exercícios musculares anaeróbicos realizados com e sem o uso de meia elástica.

\section{Métodos}

O presente trabalho está de acordo com a Resolução n ${ }^{\circ}$ 196/96 do Conselho Nacional de Saúde, de 10 de outubro de 1996.

Foram avaliadas dez jogadoras profissionais de voleibol de um mesmo time, nenhuma delas tinha o momento lesão muscular, pois isso descartaria a participação no trabalho. Tais jogadoras tinham entre 18 e 25 anos, estavam com e sem meia elástica (Sport Active ${ }^{\circledR}$, Venosan, Abreu e Lima, Brasil), com compressão de 20 a $30 \mathrm{mmHg}$ abaixo do joelho (Figura 1).

As dosagens dos biomarcadores musculares foram feitas em sangue venoso periférico em três momentos (M0, M1 e M2). O momento M0 foi sempre no início da manhã, com as atletas em repouso e sem o uso da meia elástica. $\mathrm{O}$ momento M1 foi após as jogadoras vestirem suas meias elásticas, também no início da manhã, e realizarem exercícios de acordo com o seguinte protocolo: oito minutos de alongamento; oito minutos de aquecimento composto de movimentos específicos do esporte; seis sprints de $30 \mathrm{~m} \mathrm{em}$ velocidade máxima, com 15 segundos de intervalo entre cada um, sendo $9 \mathrm{~m}$ de frente, $3 \mathrm{~m}$ de costas, $6 \mathrm{~m}$ de frente, $3 \mathrm{~m}$ de costas e $9 \mathrm{~m}$ de frente, utilizando as demarcações da quadra de voleibol. O momento M2 foi realizado sete dias depois, após o mesmo protocolo de exercícios, porém sem o uso da meia elástica. As atletas foram orientadas a

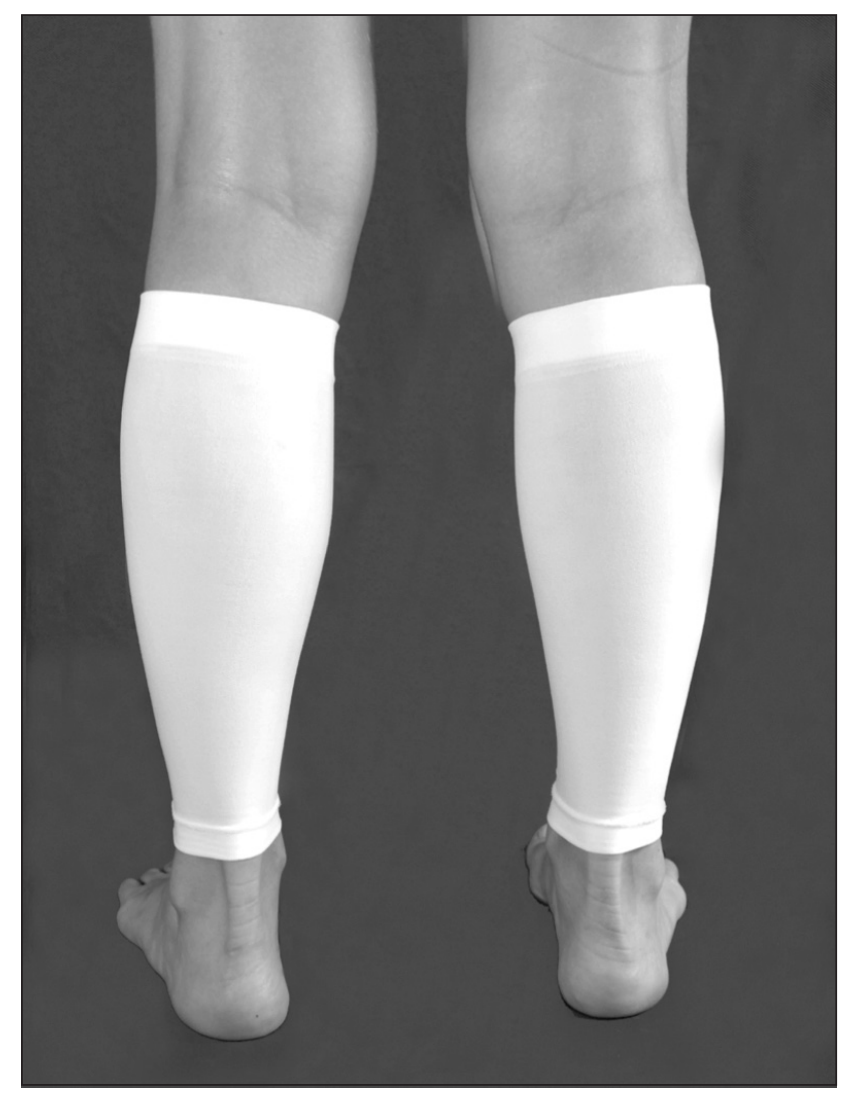

Figura 1. Meia elástica de compressão de 18 a 23 mmHg abaixo do joelho.

permanecer em repouso durante os sete dias antes do M1 e também durante os sete dias entre os M1 e M2. A escala de Borg $^{5}$ foi aplicada após duas séries de atividade física para avaliar esforço.

A análise de normalidade da distribuição dos valores das variáveis consideradas foi feita pelo teste de Shapiro-Wilk. Os níveis de CK, LDH e MB nos diferentes momentos foram comparados utilizando análise de variância (ANOVA) e pós-teste de Tukey. Os escores da escala de Borg foram comparados, utilizando-se o teste de Wilcoxon.

\section{Resultados}

As atletas participaram das três fases do estudo. Os valores de $\mathrm{CK}$, LDH e MB nos três momentos avaliados passaram no teste de normalidade de Shapiro-Wilk. Os valores médios de CK e LDH foram, respectivamente, 117,7 $\pm 40,2$ e $134,2 \pm 11,3 \mathrm{U} / \mathrm{L}$, em M0; 138,2 $\pm 47,2$ e $157,9 \pm 10,1 \mathrm{U} / \mathrm{L}$, em M1 (após atividade física com uso de meia elástica); e 161,3 $\pm 59,9$ e 177,2 $\pm 18,8$ U/L, em M2 (após atividade física sem uso de meia elástica). Os valores médios de $\mathrm{MB}$ foram de $31,5 \pm 6,5 ; 34,9 \pm 5,6$ e $38,6 \pm 12,6 \mu \mathrm{g} / \mathrm{L}$ nos momentos M0, 
M1 e M2, respectivamente. Foram observadas diferenças estatisticamente significantes entre M1 e M2 (teste de Tukey) para os valores de CK ( $\mathrm{p}=0,0007)$ e LDH $(\mathrm{p}=0,0000)$, mas não para os valores de $\mathrm{MB}(\mathrm{p}=0,1135)$. A Figura 2 ilustra os resultados obtidos nos diferentes momentos para os três biomarcadores avaliados.

Os escores médios obtidos na escala de Borg em M1 e M2 foram, respectivamente, de 17,8 $\pm 0,74$ ( $\mathrm{n}=10$, mínimo de 16 , mediana de 18 e máximo de 19) e 18,2 $\pm 0,84(n=10$, mínimo de 17 , mediana de 18 e máximo de 20 ), sem diferença estatisticamente significante entre os dois momentos $(p=0,3016)$.

\section{Discussão}

Os benefícios do uso da meia elástica na prática esportiva têm sido objeto de vários estudos. Armstrong ${ }^{11}$, por exemplo, sugere que o mecanismo responsável pela dor muscular pós-exercício é a força mecânica da contratilidade das fibras elásticas, resultando em trauma estrutural da célula. O sarcolema é lesado, levando à quebra da homeostase do cálcio, processo este que é acompanhado por necrose celular. A presença de resíduos celulares e de células imunes, por sua vez, leva à inflamação, caracterizada por edema e dor muscular. $\mathrm{O}$ uso da meia elástica tem como objetivo diminuir ou amenizar tal quadro de lesão.

Os métodos de avaliação do uso de meia elástica na prática esportiva são muito variados ${ }^{3,4,6,10}$. Neste estudo, a avaliação foi feita pela dosagem dos níveis plasmáticos de $\mathrm{CK}, \mathrm{LDH}$ e MB. O aumento dos níveis plasmáticos desses biomarcadores musculares após atividade física intensa está associado à ocorrência de lesão e, consequentemente, do sofrimento muscular ${ }^{4}$.

Os três biomarcadores avaliados no presente estudo desempenham papéis importantes na geração de

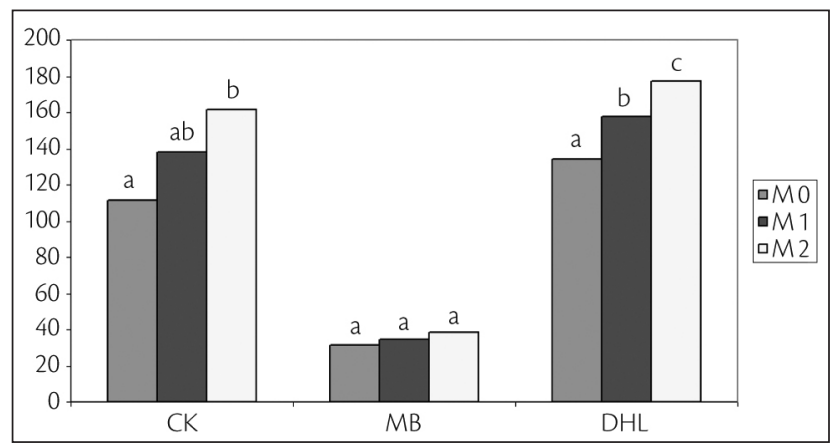

Figura 2. Valores médios obtidos para creatina quinase (CK), lactato desidrogenase (LDH) e mioglobina (MB) nos três momentos de avaliação (M0, M1 e M2). energia via metabolismo muscular, podendo permanecer elevados por até quatro dias após a atividade física inten$\mathrm{sa}^{11}$. Considerando que a magnitude dessa elevação varia de acordo com a intensidade e a frequência da atividade e com a idade e o sexo do praticante, entre outros fatores, no presente estudo, utilizou-se um grupo de atletas do mesmo sexo e com idades bem próximas, avaliado em dois momentos distintos, separados por um intervalo de sete dias, com cada atleta sendo referencial de si mesma quanto ao uso da meia elástica. Esses parâmetros foram definidos com o objetivo de evitar a interferência de qualquer fator externo na avaliação dos resultados obtidos.

Os níveis plasmáticos dos biomarcadores de lesão muscular, $\mathrm{CK}, \mathrm{LDH}$ e $\mathrm{MB}$ em M0, foram significantemente menores do que os níveis obtidos tanto em M1 quanto em M2, o que reflete os valores estacionários sanguíneos dessas biomoléculas no sangue das atletas em M0.

Por outro lado, os menores níveis de $\mathrm{CK}$ e $\mathrm{LDH}$ em M1 em relação ao M2 sugerem que o uso da meia elástica com compressão de 18 a $23 \mathrm{mmHg}$ foi associado a menores graus de lesão muscular em treinamento de alta intensidade. É possível afirmar que o resultado observado não deve ser decorrente de diferenças na carga de esforço da atividade física entre os dois momentos, pois não houve diferença estatisticamente significativa entre os escores da escala de Borg alcançados pelas atletas nas duas ocasiões. Além disso, os maiores níveis plasmáticos observados em M2 também não devem ser decorrentes de uma eventual contribuição dos níveis provenientes de M1, uma vez que quatro dias após a realização de atividade intensa já há retorno dos biomarcadores aos níveis estacionários normais das atividades enzimáticas no sangue ${ }^{11}$.

Os resultados obtidos no presente trabalho corroboram os achados de Kraemer et al. ${ }^{7}$, os quais relataram valores menores de atividade plasmática de CK com o uso de meia elástica, e também os observados por Ali et al. ${ }^{3}$, em estudo que demonstrou que o uso de meia de compressão de 18 a $23 \mathrm{mmHg}$ em corredores de $10 \mathrm{~km}$ promoveu melhora da dor e fadiga 24 horas após a atividade. O mecanismo responsável por essa melhora observada por Ali et al. ${ }^{3}$ provavelmente está relacionado com a diminuição dos níveis de $\mathrm{CK}$ e $\mathrm{LDH}$, do trauma estrutural no músculo e/ou da isquemia localizada ${ }^{3}$.

A busca por uma melhor qualidade de vida, incluindo a prática regular de atividade física, aumentou nas últimas décadas. A meia elástica aplicada à prática esportiva tem o intuito de melhorar o conforto do atleta e dos praticantes de atividade física em geral. Apesar de ainda não haver grandes estudos demonstrando os benefícios do uso da meia 
durante o exercício físico, o presente estudo sugere a ocorrência de menores níveis de lesão muscular após atividade física de alta intensidade em atletas de voleibol, a extrapolação para outros tipos de atividade física seria especulativa.

Neste estudo-piloto, a amostra foi pequena e naturalmente os resultados não consolidam a indicação deste tipo de meia para todas as atividades físicas. Outros estudos em diferentes tipos de modalidade física devem ser realizados para conclusões mais seguras.

\section{Referências}

1. Partsch H, Flour M, Smith PC. Indications for compression therapy in venous and lymphatic disease consensus based on experimental data and scientific evidence. Under the auspices of the IUP. Int Angiol. 2008;27:193-219.

2. Simao R, Farinatti Pde T, Polito MD, Maior AS, Fleck SJ. Influence of exercise order on the number of repetitions performed and perceived exertion during resistance exercises. J Strength Cond Res. 2005; 19:152-6.

3. Ali A, Caine MP, Snow BG. Graduated compression stockings: physiological and perceptual responses during and after exercise. J Sports Sci. 2007;25:413-9.

4. Berry MJ, McMurray RG. Effects of graduated compression stockings on blood lactate following an exhaustive bout of exercise. Am J Phys Med. 1987;66:121-32.

5. Borg GA. Psychophysical bases of perceived exertion. Med Sci Sports Exerc. 1982;14:377-81.

6. Kraemer W, Bush J, Bauer J, Triplett-McBride NT, Paxton NJ, Clemson A, et al. Influence of compression garments on vertical jump performance in NCAA Division I volleyball players. J Strength Cond Res. 1996;10:180-3.
7. Kraemer WJ, Bush JA, Newton RU, Duncan ND, Volek JS, Denegar $\mathrm{CR}$, et al. Influence of a compression garment on repetitive power output production before and after different types of muscle fatigue. Sports Med Train Rehabil. 1998;8:163-84.

8. Kraemer WJ, Bush JA, Wickham RB, Denegar CR, Gómez AL, Gotshalk LA, et al. Continuous compression as an effective therapeutic intervention in treating eccentric-exercise-induced muscle soreness. J Sport Rehabil. 2001;10:11-3.

9. Doan BK, Kwon YH, Newton RU, Shim J, Popper EM, Rogers RA, et al. Evaluation of a lower-body compression garment. J Sports Sci. 2003;21:601-10.

10. Bringard A, Perrey S, Belluye N. Aerobic energy cost and sensation responses during submaximal running exercise--positive effects of wearing compression tights. Int J Sports Med. 2006;27:373-8.

11. Armstrong RB. Mechanisms of exercise-induced delayed onset muscular soreness: a brief review. Med Sci Sports Exerc. 1984;16:529-38.

Correspondência Marcondes Figueiredo Rua Marques Póvoa, 88 - Martins CEP 38400-438 - Uberlândia (MG), Brasil E-mail:drmarcondes@gmail.com

Contribuições dos autores Concepção e desenho do estudo: MF. Análise e interpretação dos dados: MF, NPS.

Coleta de dados: MF, MFF. Redação do artigo: MF, NPS. Revisão crítica do texto: MF, MFF, NPS. Aprovação final do artigo*: MF, MFF, NPS. Análise estatística: NPS.

Responsabilidade geral pelo estudo: MF.

* Todos os autores leram e aprovaram a versão final submetida ao I Vasc Bras. 